\title{
Rapid detection of intestinal carriage of Klebsiella pneumoniae producing KPC carbapenemase during an outbreak
}

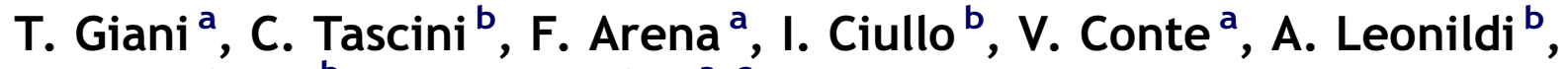 \\ F. Menichetti ${ }^{b}$, G.M. Rossolini ${ }^{a, c, *}$ \\ ${ }^{a}$ Department of Biotechnologies, Section of Microbiology, University of Siena, Siena, Italy \\ b Infectious Diseases Unit, Pisa University Hospital, Pisa, Italy \\ c Clinical Microbiology and Virology Unit, Siena University Hospital, Siena, Italy
}

\section{A R T I C L E I N F O}

\section{Article history:}

Received 16 November 2011

Accepted 1 April 2012

Available online 3 May 2012

\section{Keywords:}

Intestinal carriage

Klebsiella pneumoniae

KPC carbapenemase

Screening

\begin{abstract}
$S U M M A R Y$
Two different approaches are described for rapid detection of intestinal carriage of Klebsiella pneumoniae producing KPC-type carbapenemase (KPC-KP), based on PCR amplification of DNA extracts from rectal swabs (K-PCR), and on direct plating of rectal swabs on to MacConkey agar with a meropenem disc and a meropenem plus 3-aminophenylboronic acid disc (direct KPC screening test, DKST). K-PCR and DKST were tested with a total of 101 samples from 65 patients, during an outbreak. Although less sensitive, DKST could detect high-level carriage, which appears to be common among infected and colonised patients, while being very cheap and easy to perform, and requiring only basic facilities.
\end{abstract}

(C) 2012 The Healthcare Infection Society. Published by Elsevier Ltd. All rights reserved.

\section{Introduction}

Klebsiella pneumoniae strains producing KPC-type carbapenemases (KPC-KP) are rapidly spreading worldwide. ${ }^{1}$ Often, KPC-KP strains are multi- or extremely drug-resistant and therapeutic options are very limited. ${ }^{2}$ A higher mortality rate has been observed among patients infected with KPC-KP in comparison with those infected by antibiotic-susceptible K. pneumoniae. $^{3}$

Carriage of KPC-KP may precede infection, and carriers represent an important reservoir for dissemination of KPC-KP in the hospital setting. ${ }^{4}$ Prompt identification of carriers is a key step in effective infection control, since the control measures

\footnotetext{
* Corresponding author. Address: Department of Biotechnologies, Section of Microbiology, University of Siena, Policlinico Santa Maria alle Scotte, 53100 Siena, Italy. Tel.:+39 0577233870.

E-mail address: rossolini@unisi.it (G.M. Rossolini).
}

to reduce cross-transmission of KPC-KP in healthcare settings are mostly based on contact precautions and cohorting of all patients infected or colonised by these strains. ${ }^{5}$

In this article we describe the use of two approaches for rapid detection of carriage of KPC-KP among hospitalised patients during an outbreak.

\section{Methods \\ Detection of $K P C-K P$ in rectal swabs \\ Two methods were used for the detection of KPC-producing strains in rectal swabs: (i) a polymerase chain reaction (K-PCR) designed to detect the presence of bla $a_{\mathrm{KPC}}$ genes with high sensitivity; (ii) a culture-based, direct KPC screening test, (DKST), designed to detect KPC-KP when it represents a dom- inant fraction of the gut flora.}


The K-PCR method was carried out using primers and conditions previously described, using as template $5 \mu \mathrm{L}$ of a crude DNA extract prepared by eluting the rectal swab in $500 \mu \mathrm{L}$ of sterile normal saline for $20 \mathrm{~min}$ at room temperature, and then heating the eluted suspension at $100{ }^{\circ} \mathrm{C}$ for $15 \mathrm{~min}$ in a screw-capped vial. ${ }^{6}$

The DKST was carried out by inoculating the rectal swab directly on to a McConkey agar plate to obtain semiconfluent growth. Two discs, one containing meropenem (MER, $10 \mu \mathrm{g})$ and the other containing MER $(10 \mu \mathrm{g})$ plus 3-aminophenylboronic acid (APB, $600 \mu \mathrm{g}$, added to the carbapenem disc immediately before use) were placed onto the inoculated plate (Figure 1). Antibiotic discs were supplied by DID diagnostics, Milan, Italy. APB (Sigma Chemical Co., St Louis, MO, USA) was dissolved in dimethylsulphoxide at a concentration of $100 \mathrm{mg} / \mathrm{mL}$, and stored in the dark at room temperature until use. Plates were read after overnight incubation at $37^{\circ} \mathrm{C}$. A positive DKST was defined as lactose-fermenting, mucoid bacterial growth on the plate with no or measurable inhibition zone around the MER disc (maximum: $25 \mathrm{~mm}$ in diameter; mean: $14.3 \pm 4.4 \mathrm{~mm}$, with the studied isolates), and $\mathrm{a} \geq 5 \mathrm{~mm}$ larger inhibition zone around the MER-APB disc (Figure 1 and data not shown). A negative DKST had either a lactosefermenting or non-fermenting bacterial growth with larger inhibition zones (range: 11-38 $\mathrm{mm}$ in diameter; mean: $28.6 \pm 4.4 \mathrm{~mm}$ ), and with no or only slightly enlargement $(<5 \mathrm{~mm}$ in diameter) around the MER-APB disc.

\section{Patients and samples}

Carriage of KPC-producing strains was investigated by the two methods among inpatients from three different healthcare facilities, including one tertiary care teaching hospital, one secondary care district hospital and one rehabilitation facility, that had experienced an outbreak of KPC-KP since July 2010. Overall, during the period August-November 2010, 65 patients were investigated, including seven patients diagnosed with an infection by KPC-KP (bloodstream infection, $N=3$;

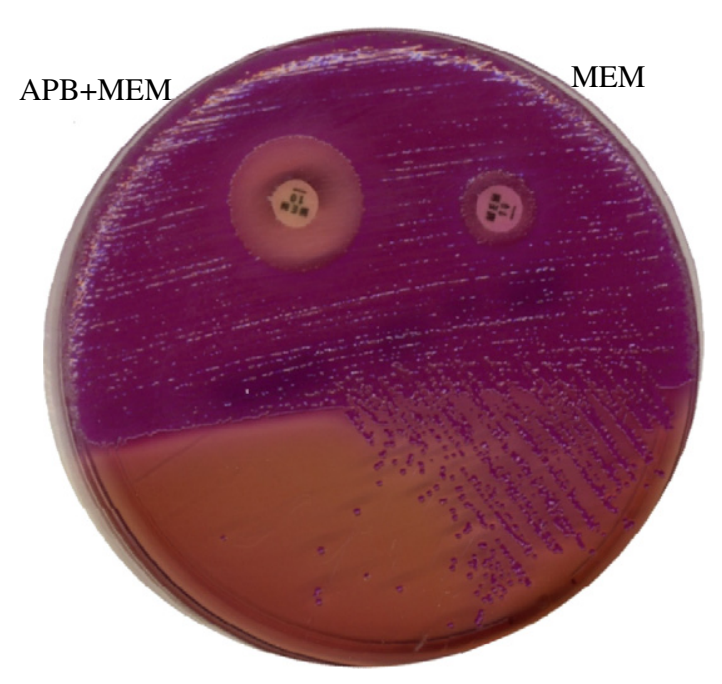

Figure 1. Appearance of a positive direct KPC screening test (DKST) test. APB, 3-aminophenylboronic acid $600 \mu \mathrm{g} ; \mathrm{MEM}$, meropenem $10 \mu \mathrm{g}$. Inhibition zone diameters were: MEM $5 \mathrm{~mm}$; $M E M+$ APB $20 \mathrm{~mm}$. bloodstream infection plus skin and soft tissue infection, $N=2$; urinary tract infection plus lower respiratory tract infection, $N=1$; complicated intra-abdominal infection, $N=1$ ) and 58 patients with no diagnosed KPC-KP infection but hospitalised in wards where at least one KPC-KP isolate had been previously reported. From each screened patient, rectal swabs were collected in duplicate and processed in parallel using the K-PCR and the DKST methods. With some infected or colonised patients, multiple rectal swabs were collected during the admission period, to follow up the carriage status over time. Informed consent was obtained from all patients or their legal guardians.

\section{Identification and characterisation of bacterial isolates}

Identification of bacterial isolates was carried out using the mini-API system (bioMérieux, Marcy l'Etoile, France). Detection of bla $a_{\mathrm{KPC}}$ genes in bacterial isolates, and genotyping by random amplification of polymorphic DNA (RAPD) and by multilocus sequence typing (MLST) was carried out as described previously. $^{6}$ Sequence data for MLST assignment were analysed using tools available on the K. pneumoniae MLST web site (http://www.pasteur.fr/recherche/genopole/PF8/mlst/ Kpneumoniae.html).

\section{Results}

\section{Detection of KPC-KP carriage by K-PCR and DKST}

K-PCR and DKST were used to detect the presence of KPC-KP in a total of 101 rectal swabs from 65 patients. Overall, 53 rectal swabs were positive with K-PCR and 46 of them (87\%) were also positive with DKST. No sample was positive with DKST and negative with K-PCR.

In all cases of DKST positivity, the lactose-fermenting and mucoid bacterial growth was identified as K. pneumoniae, and characterisation of the isolates by PCR confirmed the presence of a bla $a_{\mathrm{KPC}}$ gene. RAPD genotyping of KPC-KP revealed identical profiles, indicating that all isolates were clonally related (data not shown). MLST analysis, carried out with three randomly selected isolates from different patients, revealed that they belonged to sequence type ST258.

\section{Carriage of KPC-KP by infected and non-infected patients, and over time}

In all, 21 rectal swabs were collected from seven infected patients. These samples always yielded a positive K-PCR and DKST result, revealing a high-level intestinal carriage of KPCKP. Multiple rectal swabs collected from five of these patients revealed, in all cases, the persistence of high-level carriage of KPC-KP over time (up to 40 days) (Figure 2).

A total of 80 rectal swabs were collected from 58 noninfected patients. These samples yielded either a negative result with both tests $(N=48)$ suggesting the lack of carriage of KPC-KP, or a positive K-PCR but negative DKST result $(N=7)$ indicating a low-level KPC-KP carriage, or a positive result with both tests $(N=25)$ revealing a high-level KPC-KP carriage. Multiple rectal swabs collected from 12 carriers revealed 


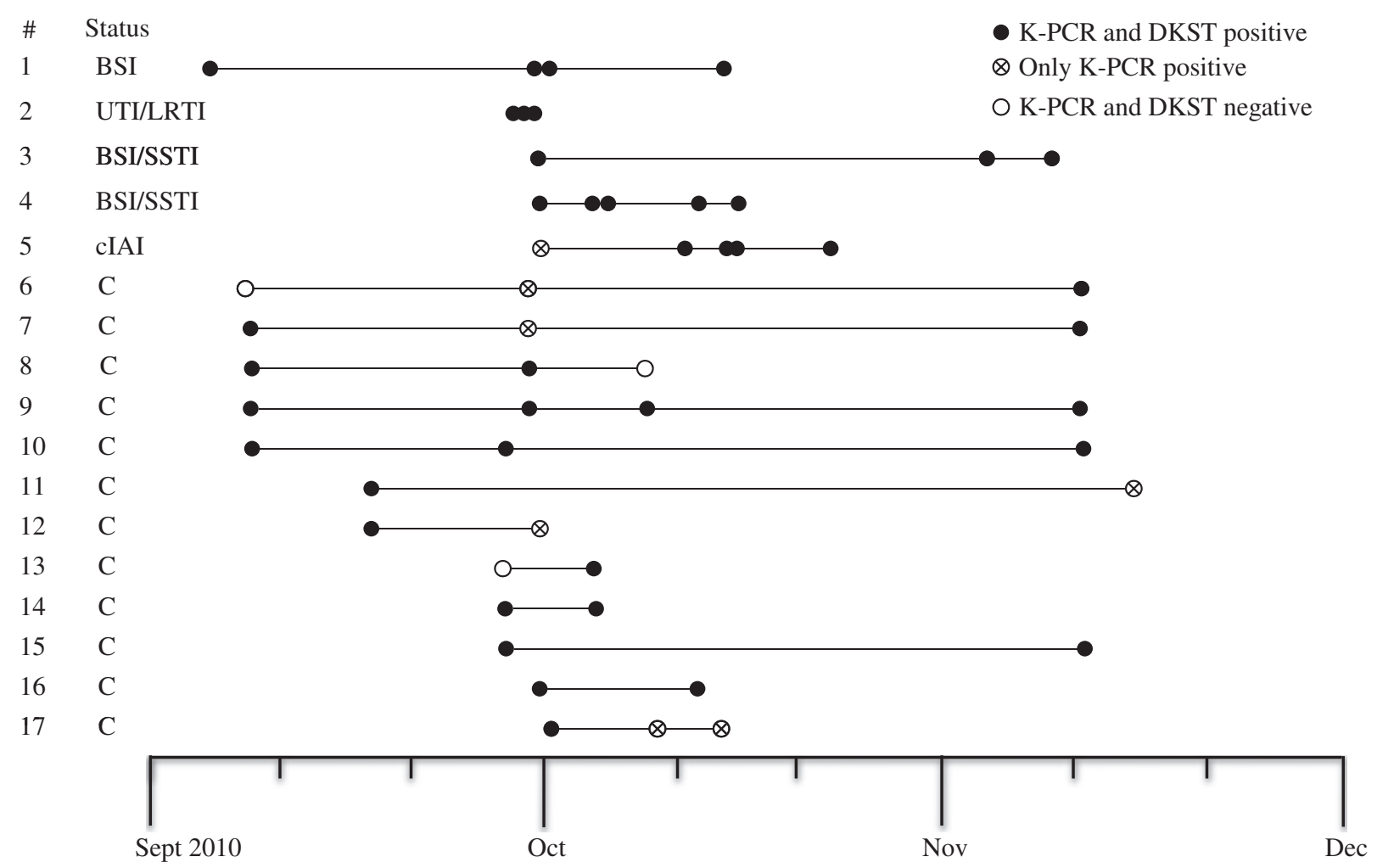

Figure 2. Detection of KPC-KP carriage in infected and in colonised patients. Only patients with replicated samples were reported. A DKST-positive result indicated high-level intestinal colonisation by KPC-KP. \#, patient number. For infected patients, types of infections are also reported. C, colonised; BSI, bloodstream infection; UTI, urinary tract infection; LRTI, lower respiratory tract infection; cIAI, complicated intra-abdominal infection; SSTI, skin and soft tissue infection.

variable patterns, including cases of prolonged high-level carriage over time (up to 64 days) (Figure 2).

\section{Discussion}

Detection of carriage of KPC-KP during outbreaks is a key step of infection control. In this study we used two different approaches for rapid detection of intestinal carriage of KPC-KP during an outbreak.

The K-PCR method, based on PCR amplification of crude DNA extracts from rectal swabs, exhibited a higher sensitivity and could provide results within $3-4 \mathrm{~h}$, with the advantage of being cheaper than real-time PCR methods previously described. ${ }^{7}$

The DKST method, based on direct plating of rectal swabs on to McConkey agar in the presence of a MER and of a MER plus APB disc (DKST), exhibited a lower sensitivity and provided results after a somewhat longer time $(16-20 \mathrm{~h})$. Since DKST was designed to detect carriage of KPC-KP when it represents a dominant fraction of the Gram-negative facultative aerobic flora of the gut, the lower sensitivity was not surprising and probably reflected a low-level carriage of KPC-KP in some cases. In our experience, a high-level intestinal carriage of KPC-KP was very frequent, being observed with all KPC-KPinfected patients and also with most colonised patients (78\%). In several cases, it was found to persist for some weeks. These findings suggest that a high-level and prolonged carriage of KPC-KP may be a common condition among hospitalised patients during outbreaks, with consequent implications for infection control practices. This high-level prolonged carriage of KPC-KP could be promoted by antimicrobial exposure and/or by biological features of the KPC-KP clone, and will be the subject of further investigation. It will also be interesting to verify whether heavily colonised patients are at higher risk of developing a KPC-KP infection. This information would be useful to guide clinical decisions in terms of delaying surgical procedures, using invasive medical devices, choosing antimicrobial prophylaxis or attempting gut decolonisation.

PCR-based methods for detection of KPC-KP carriage performed directly from clinical samples, such as the K-PCR described here, or others previously described, have the advantage of rapidity and high sensitivity, but are relatively expensive and require a suitably equipped laboratory with expertise in diagnostic molecular biology. ${ }^{7,8}$ On the other hand, culture-based methods for detection of KPC colonisation, such as DKST, can be carried out with basic laboratory facilities. Compared with other culture-based methods, DKST may exhibit a lower sensitivity but is considerably more rapid, less labour intensive, and provides information about the mechanism involved in resistance. ${ }^{9-12}$ The latter feature is an advantage of DKST also in comparison with the screening method on MacConkey agar with ertapenem disc proposed by Calfee et al., and with chromogenic media (e.g. CHROMagarTM $\mathrm{KPC}$ ) that, although highly sensitive, are unable to differentiate between KPC-producing strains and strains producing other carbapenemases, and can also return false-positive results in case of ESBL or AmpC enzyme production plus porin loss. ${ }^{10-12}$ Moreover, chromogenic media are also considerably more expensive than DKST (about eight-fold, considering 
CHROMagar KPC). DKST therefore appears to be a rapid method useful to detect carriage of KPC-KP, which may also be performed by small microbiology laboratories in resource-limited settings.

The finding that all isolates belonged to the same clonal lineage (ST258) confirmed the ability of this successful clone to spread between different hospital settings worldwide. ${ }^{1}$

\section{Conflict of interest statement}

None declared.

\section{Funding sources}

This work was partially supported by funding from the European Commission under the 7th Framework Programme (TROCAR contract HEALTH-F3-2008-223031, TEMPOtest-QC contract HEALTH-2009-241742 and EvoTAR contract HEALTH-F3-2011-282004).

\section{References}

1. Nordmann P, Naas T, Poirel L. Global spread of carbapenemase-producing Enterobacteriaceae. Emerg Infect Dis 2011;17:1791-1798.

2. Hirsch EB, Tam VH. Detection and treatment options for Klebsiella pneumoniae carbapenemases (KPC): an emerging cause of multidrugresistant infection. J Antimicrob Chemother 2010;65:119-125.

3. Patel G, Huprikar S, Factor SH, Jenkins SG, Calfee DP. Outcomes of carbapenem-resistant Klebsiella pneumoniae infection and the impact of antimicrobial and adjunctive therapies. Infect Control Hosp Epidemiol 2008;29:1099-1106.
4. Agodi A, Voulgari E, Barchitta M, et al. Containment of an outbreak of KPC-3-producing Klebsiella pneumoniae in Italy. J Clin Microbiol 2011;49:3986-3989.

5. Miriagou V, Cornaglia G, Edelstein $M$, et al. Acquired carbapenemases in gram-negative bacterial pathogens: detection and surveillance issues. Clin Microbiol Infect 2010;16:112-122.

6. Giani T, D'Andrea MM, Pecile P, et al. Emergence in Italy of Klebsiella pneumoniae sequence type 258 producing $\mathrm{KPC}-3$ carbapenemase. J Clin Microbiol 2009;47:3793-3794.

7. Mangold KA, Santiano K, Broekman R, et al. Real-time detection of $b / a_{\mathrm{KPC}}$ in clinical samples and surveillance specimens. J Clin Microbiol 2011;49:3338-3339.

8. Schechner V, Straus-Robinson K, Schwartz D, et al. Evaluation of PCR-based testing for surveillance of KPC-producing carbapenemresistant members of the Enterobacteriaceae family. J Clin Microbiol 2009;47:3261-3265.

9. Landman D, Salvani JK, Bratu S, Quale J. Evaluation of techniques for detection of carbapenem-resistant Klebsiella pneumoniae in stool surveillance cultures. J Clin Microbiol 2005;43: 5639-5641.

10. Calfee D, Jenkins SG. Use of active surveillance cultures to detect asymptomatic colonization with carbapenem-resistant Klebsiella pneumoniae in intensive care unit patients. Infect Control Hosp Epidemiol 2008;29:966-968.

11. Panagea T, Galani I, Souli M, Adamou P, Antoniadou A, Giamarellou H. Evaluation of CHROMagar ${ }^{\mathrm{TM}}$ KPC for the detection of carbapenase-producing Enterobacteriaceae in rectal surveillance cultures. Int J Antimicrob Agents 2011;37:124-128.

12. Adler A, Navon-Venezia S, Moran-Gilad J, Marcos E, Schwartz D, Carmeli Y. Laboratory and clinical evaluation of screening agar plates for detection of carbapenem-resistant Enterobacteriaceae from surveillance rectal swabs. J Clin Microbiol 2011;49: 2239-2242. 